Session 3230

\title{
Preliminary Results from the Development of a Concept Inventory in Thermal and Transport Science
}

\author{
Barbara M. Olds ${ }^{1}$, Ruth A. Streveler, Ronald L. Miller \\ Colorado School of Mines
}

\author{
Mary A. Nelson \\ University of Colorado-Boulder
}

\begin{abstract}
This paper reports on the progress made in the creation of a concept inventory in thermal and transport science. We discuss the steps taken to create questions for the concept inventory and the results of the alpha testing of our inventory. Next steps in the process particularly establishing validity and reliability of the instrument - are also discussed.

\section{Introduction}

Engineering faculty members often comment that even students who can correctly solve problems in fluid dynamics, heat transfer, or thermodynamics still mistakenly believe that, for example, heat flows like a substance or that processes stop when they reach equilibrium. These observations are supported by evidence in the literature that suggests that engineering and science students often have fundamental misconceptions about the way that molecular-scale processes differ from observable, macroscopic causal behavior we experience in our daily lives.
\end{abstract}

To help faculty identify the concepts that their students do not understand and decide which misconceptions are most prevalent, a number of instruments, called concept inventories or CI's, have been developed in selected fields, most notably the Force Concept Inventory in physics. With NSF support, we are developing a concept inventory for thermal and transport sciences encompassing introductory thermodynamics, fluid mechanics, and heat transfer. At ASEE 2003 we reported on the results of a Delphi study we conducted with approximately 30 engineering faculty experts to reach consensus about the difficulty and importance of fundamental concepts in the thermal and transport sciences. Based on the results of that study, we have identified key concepts and developed the alpha version of our concept inventory. In this paper we will describe the development and testing of the alpha version concept inventory, present our preliminary analysis of the results from the alpha test, and discuss our plans for assuring the validity and reliability of our finished $\mathrm{CI}$.

\footnotetext{
${ }^{1}$ Contact author: Barbara M. Olds, Professor of Liberal Arts and International Studies, Colorado School of Mines, Golden, CO 80401, bolds@mines.edu, (703) 292-4429. 


\section{Background}

There is a large and growing body of research on misconceptions (or "alternate conceptions"). ${ }^{1}$ The topic has garnered considerable interest among engineering educators over the past few years and several concept inventories on engineering-related topics are being developed, most notably by the group led by Evans associated with the Foundation Coalition. ${ }^{2}$ The goal of our project, funded by the Assessment of Student Achievement (ASA) program at NSF, is to develop and test an inventory for the thermal and transport sciences, based on the model of the Force Concept Inventory pioneered by Hestenes and colleagues. ${ }^{3}$ Once our CI has been developed and validated, it will be made available to interested engineering faculty for use as a classroom formative assessment tool that can provide valuable information for tracking student understanding and developing curriculum.

As the first step in developing the CI, we conducted a Delphi study with approximately 30 experienced engineering faculty experts (people with at least five years of teaching experience and involvement in the engineering education community) and prominent engineering textbook authors to identify important concepts in thermal and transport science disciplines that are consistently difficult for students to understand and for which students possess significant and robust misconceptions. ${ }^{4}$ Based on their preliminary feedback, we developed a list of 28 concepts that we asked the experts to rate in two ways - the degree to which undergraduate engineering students understand each concept and how important the concept is for students to know. Based on these results we developed a list of 10 items that the study participants judged to be sufficiently important to engineering graduates that conceptual understanding is crucial but often not achieved. These 10 items include key topics such as the $2^{\text {nd }}$ law of thermodynamics including reversible vs. irreversible processes, conservation of momentum, viscous momentum transfer, several energy-related topics (heat, temperature, enthalpy, internal energy), and steady-state vs. equilibrium processes.

\section{Developing the Concept Inventory}

Using the ten concepts we identified through the Delphi study, we constructed sample questions and then conducted "think aloud" sessions with six Colorado School of Mines students (half chemical engineering and half mechanical engineering majors, two females and four males), asking each of them to answer three or four questions, explaining their thought processes out loud as they did so. The students were volunteers who were selected on a first-come basis and compensated for their time. We followed a protocol during the sessions: first we gave the student a written copy of each question and then read it aloud. We asked the student to tell us what concept $\mathrm{s} /$ he thought the question addressed and then asked him or her to solve the problem thinking aloud and using sketches, notes, etc., if desired. We asked questions intended to probe the students' thought processes as they solved the problems and tape-recorded the sessions for later transcription and analysis. We also collected all notes and equations the students produced. Based on these interviews, we hoped to accomplish two goals: 1) assure that 
the questions were clearly worded and illustrated so that students would not be confused by the framing of the questions, and 2) begin to develop distracters for our multiple choice concept inventory.

As an example, Table 1 contains one of the questions that we asked students to solve using the "think aloud" method. Table 2 includes some of the dialogue from a student interview to give a flavor for the form that the interviews took. (We obtained informed consent from all student participants and have not used their real names in reporting on the interviews.)

Question 6.A

A mass of air is contained in a rigid vessel at $100{ }^{\circ} \mathrm{C}$ and $5 \mathrm{~atm}$ pressure. An equal mass of air is flowing at the same temperature and pressure through a circular pipe.

Which of these samples of air can be used to produce more mechanical work?

Table 1: Sample "Think Aloud" Question

Interviewer: What are you looking at here if you were to describe the two things that you are being asked to look at? What are you looking at?

Diana: I'm not really sure, like, I'm just trying to think why both of them would have, uhm. I'm trying to think why one of them would produce more work than the other one. And in thinking about it I really don't think that both of them should produce different amounts of work.

Interviewer: Ok. So your initial thought on it would be that they would produce equal

Diana: yeah.

Interviewer: amounts of work. Ok. Why would you think that?

Diana: Well. Just because I don't think the work depends on the area in which the mass of air is contained.

Interviewer: Um-hum.

Diana: So, I mean if they're the same temperature and the same pressure and have the same volume so I just don't see why they would have a different amount of work.

Table 2: Excerpt from Student “Think Aloud” Interview

After completing the "think aloud" interviews, we carefully reviewed the transcripts and then revised the questions to increase clarity and developed a set of distracters for each question using misconceptions discovered during the analysis of the sessions as well as, 
whenever possible, using the exact language of the student interviewees. If students stated that they needed more information to answer a question, the question was rewritten to attempt to eliminate ambiguity. Table 3 contains the alpha test version of Question 6.A we developed using this process.

\section{Question 6.A}

A mass of air is contained in a rigid vessel at $100{ }^{\circ} \mathrm{C}$ and $5 \mathrm{~atm}$ pressure. An equal mass of air is flowing at the same temperature and pressure through a circular pipe.

Which of these samples of air can be used to produce more mechanical work?

a. Both will produce the same amount of work because they are at the same temperature and pressure.

b. Moving air will produce more work because it has kinetic energy and air in rigid vessel does not.

c. Air in rigid vessel will produce more work because moving air will lose energy as friction dissipation.

d. Moving air will produce more work because it contains flow work (pressure-volume work) and air in the rigid vessel does not. (Correct answer $=\mathrm{d}$ )

Table 3: Alpha Version of Question 6.A

\section{The Alpha Testing}

In the fall of 2003 we administered an alpha version of the concept inventory consisting of 11 multiple choice questions to 93 students in two classes at the Colorado School of Mines - 39 students in a senior-level chemical engineering course in Transport Phenomena and 54 students in a senior-level integrated laboratory course designed for students with a specialty in mechanical engineering. The alpha version of the test can be found in Appendix A. All of the students were seniors who had taken at least one course in thermodynamics, heat transfer, and fluids.

Several of the questions had two parts $(1,2,7 \mathrm{a}$ and $7 \mathrm{~b})$ in order to probe the reasons students gave for their answers. We therefore had a total of 15 separate questions. We also asked each student to provide an essay response to one of the questions for which we did not provide multiple possible answers. Seventy-one percent of the students (66) completed the inventory, which was used as an extra-credit take-home activity. Table 3 shows:

- N--the number of responses to each question

- Ans--the correct response to each question

- A-J - the number of students selecting each answer, and

- PER--the percentage of those answering the question correctly (Percent correct). 


\begin{tabular}{|l|l|l|l|l|l|l|l|l|l|l|l|l|l|}
\hline Q\# & N & Ans. & PER & A & B & C & D & E & F & G & H & I & J \\
\hline 1a & 66 & B & 39.4 & 22 & 26 & 2 & 8 & 8 & & & & & \\
\hline 1b & 65 & F & 41.5 & & & & & & 27 & 3 & 23 & 7 & 5 \\
\hline $2 \mathrm{a}$ & 65 & B & 47.7 & 22 & 31 & 2 & 10 & & & & & & \\
\hline $2 \mathrm{~b}$ & 66 & F & 37.9 & & & & & 10 & 25 & 11 & 8 & 12 & \\
\hline 3 & 66 & B & 60.6 & 2 & 40 & 23 & 1 & & & & & & \\
\hline 4 & 66 & D & 71.2 & 0 & 4 & 0 & 47 & 15 & & & & & \\
\hline 5 & 66 & A & 43.9 & 29 & 5 & 11 & 6 & 15 & & & & & \\
\hline 6 & 66 & D & 28.8 & 13 & 31 & 3 & 19 & & & & & & \\
\hline $7 \mathrm{a} 1$ & 66 & C & 65.2 & 16 & 6 & 43 & 1 & & & & & & \\
\hline $7 \mathrm{a} 2$ & 66 & F & 47 & & & & & 16 & 31 & 13 & 6 & & \\
\hline $7 \mathrm{~b} 1$ & 66 & B & 66.7 & 1 & 44 & 5 & 16 & & & & & & \\
\hline $7 \mathrm{~b} 2$ & 66 & F & 66.7 & & & & & 7 & 44 & 15 & 0 & & \\
\hline 8 & 66 & C & 56.1 & 8 & 16 & 37 & 5 & 0 & & & & & \\
\hline 9 & 66 & A & 45.5 & 30 & 12 & 9 & 9 & 6 & & & & & \\
\hline 10 & 66 & C & 65.2 & 3 & 16 & 43 & 4 & & & & & & \\
\hline
\end{tabular}

Table 3. Results of Alpha Test by Question

\section{Analysis of Results}

We have begun to analyze the results from our alpha test and plan to conduct extensive beta testing during 2004. This testing will include students from at least two other universities in addition to Mines students. One of these sites will provide a more diverse population than the student body of Mines allowing us to check for ethnic or racial bias in the CI. During beta testing, we will use the questions developed for the alpha test with some revised distracters. These new distracters will appear in questions where a high percentage of students chose the correct answer or will replace distracters which were chosen by virtually no one during the alpha testing. In addition, we are including two new questions for each concept and plan to conduct rigorous statistical analysis (described below) once we have completed the beta testing. The new questions have been reviewed by a focus group of five students and revised to eliminate concerns raised by the group.

Our analysis of results of the Fall 2003 alpha testing yielded the following preliminary conclusions:

1. Testing overall scores by group, we found:

A. by gender, there was no significant difference.

B. by major, the chemical engineering students performed significantly better in overall score. We have no firm evidence to explain this difference. One possibility is that because the primary author of the concept questions is a chemical engineer, he may have unintentionally biased the questions towards the CSM chemical engineering curriculum. We should be able to test this hypothesis 
when the beta test is administered to a larger sample of students at additional universities.

2. Testing individual questions by group, we found:

A. by gender, males did statistically better on question $2 \mathrm{a}$ than females and that females did better on questions $6,7 \mathrm{~b} 1$, and $7 \mathrm{~b} 2$.

B. by major, the chemical engineering students did statistically better on questions 4 (heat vs. internal energy), 7a1, 7b1 and 7b2 (steady state vs. equilibrium), 8 (viscous momentum flux) and 9 (ideal gas law). Some questions have distracters which a high percentage of students chose. These are highlighted in yellow on Table 3 . We need to analyze why they may have been attractive to students and explore whether they are among the common misconceptions identified by our expert panel in the Delphi study.

3. We identified some questions which more than $60 \%$ of students answered correctly with no common misconception being selected. These questions are being analyzed and if beta testing shows the same results, we will consult our Delphi experts in a second attempt to identify distracters for the questions that more adequately address the misconception that students hold.

4. Some distracters were virtually never chosen. For example, for question $7 \mathrm{~b} 1$ only one student chose distracter a. For these cases, an attempt will be made to replace the given distracter with one that better encapsulates student misconceptions.

5. For some questions, like 1a, we feel that we are identifying a common misconception that many students hold. We will further examine this hypothesis in our beta testing, and if analyses indicate this is a misconception that needs to be addressed, then our next research will examine how such a misconception can be confronted and possibly "repaired" by using computer simulations, for example.

\section{Validity and Reliability: Next Steps}

In addition to continuing to analyze our preliminary results, we have worked and will continue to work to ensure the validity and reliability of our instrument in a variety of ways, as discussed below. Although there are many types of validity to consider, we will focus, at least preliminarily, on construct, content, predictive and external validity questions.

Construct and content validity (Are we measuring what we think we are measuring and have we covered the domain of what we want to measure?). We have worked to ensure construct and content validity in the following ways:

We have consulted experts about the concepts that have been chosen. We conducted an extensive Delphi study to develop our list of most important and least understood concepts. These concepts were incorporated into our alpha test. In addition, we have consulted experts about the wording of the questions in the alpha version and when the 
beta version of the instrument is ready, we will engage our panel of approximately 30 experts to ensure that the questions are phrased and illustrated accurately and clearly.

We reviewed approximately 10 of the most popular textbooks in the areas covered by our instrument in order to gather additional information about how the difficult concepts are currently presented to students.

We examined the literature to determine which concepts have been addressed. This literature was instrumental in writing both questions and distracters once our Delphi study identified the concepts to be included in the instrument.

We also used student think-alouds to confirm that the concepts identified by the Delphi were misunderstood by students and to develop appropriate distracters for the questions. We had six students (juniors and seniors) think aloud about the answers to conceptual questions to see where they were having conceptual difficulties. These sessions consisted largely of constructed response items since they are more likely to inform us as to whether we are measuring the concepts that we intended to measure. We recorded and coded all think-alouds to ensure a complete and accurate picture of student responses. These think-alouds gave us important feedback that in some cases uncovered items that were misunderstood because of poor question construction, use of graphics, symbols, or notation. $^{5}$

In beta testing, we will insert one constructed response question for each concept we are measuring. Constructed responses help us to see if we are really measuring what we intend to measure. Responses allow us to see if the students are misunderstanding or misinterpreting the question. Structural Equation Modeling (SEM) will then allow us to check that the constructed response and multiple-choice items are measuring the same concept. (SEM techniques may be "viewed as 'fancy' multivariate regression methods." A unique contribution of SEM analysis is the ability to create latent variables that then can be used in the regression.) This will establish greater confidence in the construct validity of the multiple-choice items. The final version will contain only multiple-choice items.

Another check of construct validity is to determine if the number of concepts we believe we are testing is the actual number being tested. We need to be certain that if we think there are, say, three main concepts being tested, that there are in fact three. If not, then we have failed to measure the concepts that we think we are measuring. In order to test this, we will need to do a confirmatory factor analysis. We will do this analysis with Structural Equation Modeling (SEM) when we have a minimum of three questions developed for each concept.

Bias is also considered to be a component of construct and content validity. (Does the instrument measure different groups of the same ability differently?)

- We intend to analyze the readability of instructions and items for different groups (ethnic, gender, English-language learners) to ensure that construct irrelevant 
difficulty is minimized.

- We will test for any differences among the disciplines which might use the concept inventory.

- We will examine the extent and reasons for any low CI scores common to a given group, especially if they are high performing otherwise.

External Validity (Can we generalize this? Does it have predictive ability?) After the instrument is in use, we can conduct the following research experiments to test its external validity.

Do we get the same results for our instrument with a variety of populations? After our concept inventory has been tested on one population, we need to test it on other populations to be sure that its validity is not population dependent. Once we have developed the beta version of our CI, we will test it not only with Colorado School of Mines students, but also with other student groups from institutions that have agreed to be beta testing sites.

We will keep an item history database for each item when we begin beta testing. It will include differences in correct response rates by different groups, revisions to questions, etc.

Predictive Validity (Can this test be used to predict performance on another test, on success in a job, etc.?)

In order to determine the predictive validity of the test, we must be very clear about its purpose. Is it simply to give teachers information about student misconceptions, or is it a predictor for how well they are likely to do on the Fundamentals of Engineering (FE) exam or in their jobs? If the latter is the case, then we have to do some standard setting. What is the appropriate cut-off score for the predictions that we intend to make?

We may compare the results of our CI to results of standard engineering exams, such as the FE. Even though the concepts tested may not be the same, the FE purports to separate engineers into two groups: competent and non-competent, and we may try scaling the $\mathrm{CI}$ to measure competence.

We could also compare the results of our CI with actual job performance after students have graduated and have been in the workforce for some time. Using interviews or an employer survey, it may be possible to predict how well former students will perform based on the CI. Again scaling would be required. Survey and interview questions would focus on whether these new employees exhibit good conceptual understanding in their work.

Reliability. (Do repeated administrations of the test yield the same results?) A test cannot be valid if it is not reliable. Although we are in the early stages of 
developing our instrument and hence cannot yet test it for reliability, we plan to use a variety of reliability checks at the appropriate time. We will use test-retest methods on some subset of students to examine the stability of scores to be sure that students are not simply guessing because the test has no effect on their grade.

We will not use alternate forms of the CI to test reliability, because it would require us to develop and test an inordinate number of items. We may explore split-form reliability. Using this method, if we had four questions for each concept, we could split the test in two by randomly splitting the questions for each concept and then check to see if the scores on both forms were reliably the same. We will need to administer more tests before we can check for Cronbach's alpha to see how reliable our results are.

Once we have the results of the factor analysis discussed above, we will identify high and low discriminating items through discriminant analysis using CONFA and then select out the high discriminating items. We will also use the beta testing to eliminate distracters that are chosen by very few students and will replace them with better distracters based on further think-alouds. Table 3, which includes the number of responses to each item in our multiple-choice alpha instrument, clearly indicates that some distracters were chosen by few or no students. We will replace these before conducting the beta test.

After the CI is established, we may extend our research through a quasi-experiment in which we identify two classes of the same course and randomly assign one to be the treatment group. The treatment group will receive intensive instruction on the difficult concepts in addition to the normal material while the control group is taught with no changes to the curriculum. We would administer the $\mathrm{CI}$ at the beginning and conclusion of both classes and see if there were any significant differences in the gains of the two groups.

\section{Acknowledgment}

We acknowledge the financial support of the National Science Foundation for this work (project DUE-0127806).

\section{Bibliography}

1. Pfundt, H. \& Duit, R. (1994). Bibliography: Students'Alternative Frameworks and Science Education, $4^{\text {th }}$ edition. Kiel, Germany: Institute for Science Education.

2. Evans, D. L., "Tools for Assessing Conceptual Understanding in the Engineering Sciences," Proceedings of the Frontiers in Education Conference, Boston, MA, November 6-9, 2002.

3. Hestenes, D., Wells, M. and Swackhamer, G., "Force Concept Inventory," The Physics Teacher, 30, 159-166, 1992.

4. Streveler, R.A., Olds, B.M., Miller, R. L. \& Nelson, M.A. (June, 2003). "Using a Delphi Study to Identify the Most Difficult Concepts for Students to Master in Thermal and Transport Science. Proceedings of the Annual Conference of the American Society for Engineering Education, Nashville, TN.

5. Ericson K. A. \& Simon, H. A. (1996). Protocol Analysis, $2^{\text {nd }}$ edition. MIT Press: Cambridge, MA.

6. Mueller, R. O. (1999). Basic Principles of Structural Equation Modeling. Springer: New York. 


\section{Biographical Information}

BARBARA M. OLDS is Professor of Liberal Arts and International Studies at the Colorado School of Mines. She has participated in a number of curriculum innovation projects and has been active in the engineering education and assessment communities. She was a Fulbright lecturer/researcher in Sweden in 1999. Dr. Olds is presently serving as Director of the Division of Research, Evaluation and Communication in the EHR Directorate of the National Science Foundation.

RUTH A. STREVELER is the Director of the Center for Engineering Education at the Colorado School of Mines. Dr. Streveler received her Ph.D. in Educational Psychology from the University of Hawaii at Manoa. She also holds a Master of Science in Zoology from the Ohio State University and a Bachelor of Arts in Biology from Indiana University at Bloomington. She is co-director of an NSF-sponsored project to develop assessment instruments for identifying misconceptions in engineering students as well as coprincipal investigator for the Center for the Advancement of Engineering Education that is also funded by NSF.

RONALD L. MILLER is professor of chemical engineering at the Colorado School of Mines where he has taught chemical engineering and interdisciplinary courses and conducted research in educational methods for the past seventeen years. He has received three university-wide teaching awards and has held a Jenni teaching fellowship at CSM. He has received grant awards for educational research from the National Science Foundation, the U.S. Department of Education (FIPSE), the National Endowment for the Humanities, and the Colorado Commission on Higher Education.

MARY A. NELSON is a PhD candidate in the Research and Evaluation Methods program at the University of Colorado, Boulder working with Dr. Lorrie Shepard. Mary has taught math at the middle school, high school, and college levels for nearly 35 years and is presently conducting research on two funded grants studying how middle school students learn algebra and assessing engineering student misconceptions in thermal and transport sciences. 
Appendix I

Question 1.

Version 1.0 - Reliabilty and validity not yet determined. Not ready for general use.

For the piping system shown below, water is flowing at steady-state and constant temperature from left to right.

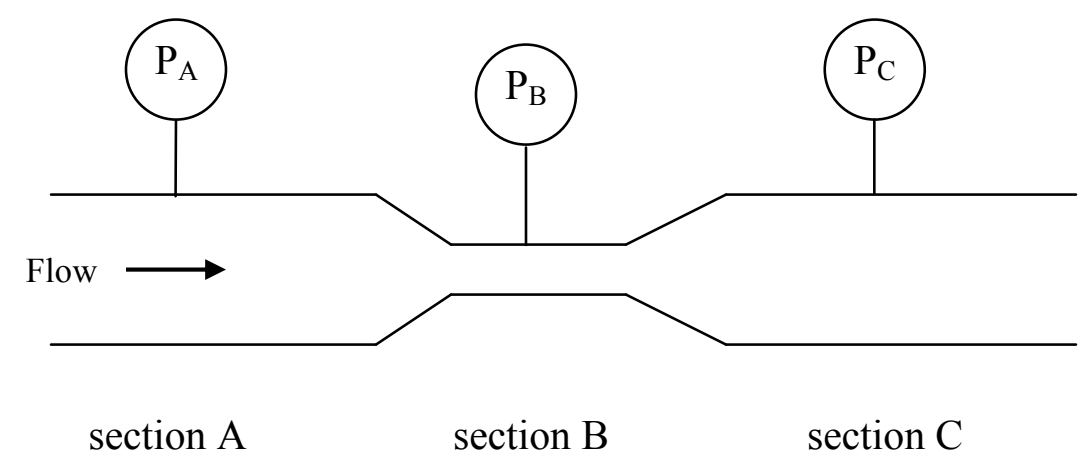

The pipe diameter is larger in section A than section B. The diameters of sections A and $\mathrm{C}$ are the same. If gravitation and frictional effects are negligible, which of the following relationships is true about the pressure in the system?
a. $\quad \mathrm{P}_{\mathrm{A}}<\mathrm{P}_{\mathrm{B}}$ and $\mathrm{P}_{\mathrm{A}}=\mathrm{P}_{\mathrm{C}}$
b. $\quad \mathrm{P}_{\mathrm{A}}>\mathrm{P}_{\mathrm{B}}$ and $\mathrm{P}_{\mathrm{A}}=\mathrm{P}_{\mathrm{C}}$
c. $\mathrm{P}_{\mathrm{A}}=\mathrm{P}_{\mathrm{B}}=\mathrm{P}_{\mathrm{C}}$
d. $\mathrm{P}_{\mathrm{A}}>\mathrm{P}_{\mathrm{B}}$ and $\mathrm{P}_{\mathrm{B}}>\mathrm{P}_{\mathrm{C}}$
e. $\mathrm{P}_{\mathrm{A}}<\mathrm{P}_{\mathrm{B}}$ and $\mathrm{P}_{\mathrm{B}}>\mathrm{P}_{\mathrm{C}}$

The answer selected above is correct because:

f. Pressure builds up when mass is being pushed through a smaller area

g. Friction is assumed to be negligible

h. Pressure decreases as velocity increases

i. Pressure drops in the direction of the flow

j. System is at steady-state 
Version 1.0 - Reliabilty and validity not yet determined. Not ready for general use.

Question 2.

For the piping system shown below, water is flowing from point 1 to 2 (left to right). The pressures at these points are $\mathrm{P}_{1}$ and $\mathrm{P}_{2}$, respectively. The velocities at these points are $\mathrm{v}_{1}$ and $\mathrm{v}_{2}$, respectively. If we want to determine the net force of the water on the pipe wall using a momentum balance on the fluid in the pipe, which of the following diagrams correctly shows the direction of velocity vector and force vector (due to pressure) acting on the fluid at the inlet and outlet of the pipe?

You may assume that the velocity is constant across the pipe cross-section.

a.

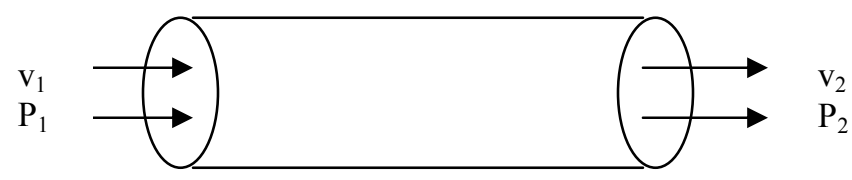

b.

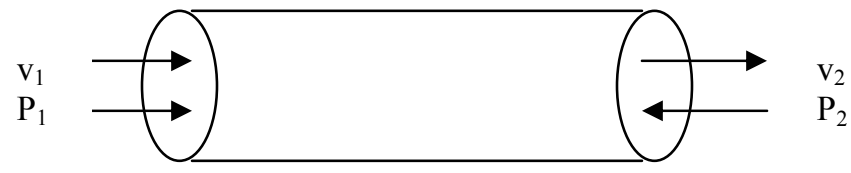

c.

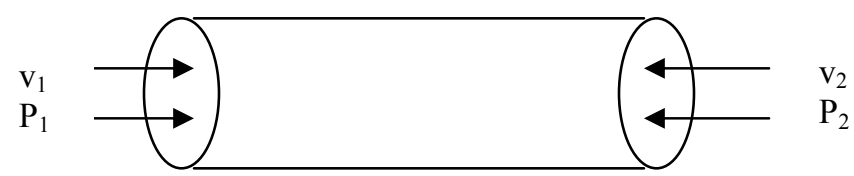

d.

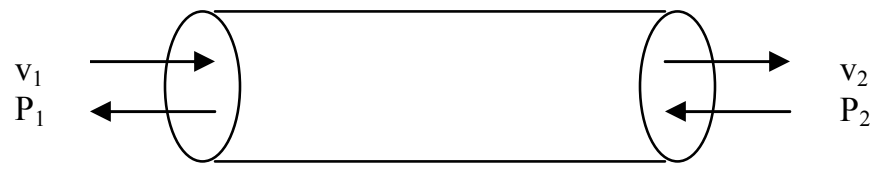

The answer selected above is correct because:

e. Direction of velocity and pressure are related

f. Pressure acts in all directions so the force vector points into pipe cross-section at entrance and exit

g. Velocity and pressure act in same direction since both terms are included in the Bernoulli equation

h. Velocity and pressure act in opposite directions since velocity increases as pressure decreases according to the Bernoulli equation

i. Pressure is trying to hold fluid in the pipe by opposing the flow 
Version 1.0 - Reliabilty and validity not yet determined. Not ready for general use. Question 3.

A small piece of metal at $75{ }^{\circ} \mathrm{C}$ is placed into an insulated beaker of water at $25{ }^{\circ} \mathrm{C}$ as shown below. After a long period of time, how would the temperatures of the water and metal bar be related?

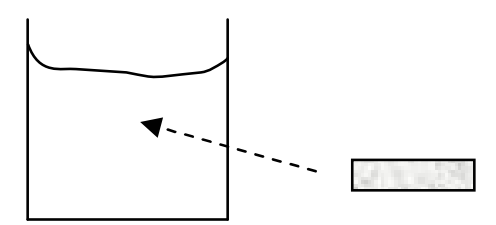

water at $25{ }^{\mathrm{O}} \mathrm{C} \quad$ metal bar at $75{ }^{\circ} \mathrm{C}$

a. Both metal and water would end up at $50{ }^{\circ} \mathrm{C}$ because this is the average of the two starting temperatures $\left(25^{\mathrm{O}} \mathrm{C}\right.$ and $\left.75^{\mathrm{O}} \mathrm{C}\right)$.

b. The temperature of the metal bar and water would be the same and would end up somewhere between $25{ }^{\circ} \mathrm{C}$ and $75^{\circ} \mathrm{C}$ depending upon the heat capacity of the metal and water.

c. The temperature of the metal bar and water would be the same and would end up somewhere between $25{ }^{\circ} \mathrm{C}$ and $75{ }^{\circ} \mathrm{C}$ depending upon the thermal conductivity of the metal and water.

d. The metal bar would always remain hotter than water because the metal has a higher thermal conductivity than water. 
Version 1.0 - Reliabilty and validity not yet determined. Not ready for general use.

Question 4.A

An engineering student walking barefoot (without shoes or socks) from a tile floor onto a carpeted floor notices that the tile feels cooler than the carpet. Which of the following explanations seems like the most plausible way to explain this observation?

a. The tile has a lower temperature than the carpet because the tile emits more black body radiation than the carpet.

b. The tile has a lower temperature than the carpet because the carpet absorbs more energy from the light in the room.

c. The tile has a lower temperature than the carpet because the carpet absorbs more energy from the air in the room.

d. The tile is a better conductor of energy than the carpet, so energy is conducted away from the student's foot faster on tile than carpet.

e. The convective heat transfer coefficients for the tile and carpet are not the same values. 
Version 1.0 - Reliabilty and validity not yet determined. Not ready for general use.

\section{Question 5.}

Two identical containers contain equal masses of liquid at $20{ }^{\mathrm{O}} \mathrm{C}$ as shown below. One container is filled with water and the other container with ethanol (ethyl alcohol). Energy is added to each container with identical Bunsen burners. After 2 minutes, the temperature of the ethanol is $40{ }^{\mathrm{O}} \mathrm{C}$. It takes 3 minutes for the water temperature to rise to $40{ }^{\mathrm{O}} \mathrm{C}$.
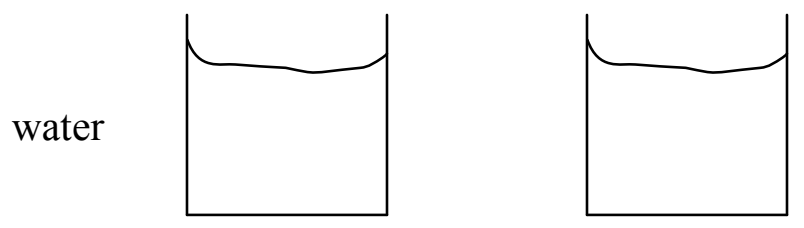

ethanol

To which liquid was more energy transferred?

a. Water because more energy is transferred to the liquid that is heated longer.

b. Alcohol because more energy is transferred to the liquid that heats up faster (temperature rises faster).

c. Both liquids received the same amount of energy because they started at the same initial temperature and ended at the same final temperature.

d. Can't determine from the information given because heat transfer coefficients for water and ethanol are needed.

e. Can't determine from the information given because heat capacities of water and ethanol are needed. 
Version 1.0 - Reliabilty and validity not yet determined. Not ready for general use.

\section{Question 6.}

A mass of air is contained in a rigid vessel at $100{ }^{\circ} \mathrm{C}$ and $5 \mathrm{~atm}$ pressure. An equal mass of air is flowing at the same temperature and pressure through a circular pipe.

Which of these samples of air can be used to produce more mechanical work?

a. Both will produce the same amount of work because they are at the same temperature and pressure.

b. Moving air will produce more work because it has kinetic energy and air in rigid vessel does not.

c. Air in rigid vessel will produce more work because moving air will lose energy as friction dissipation.

d. Moving air will produce more work because it contains flow work (pressurevolume work) and air in the rigid vessel does not. 
Version 1.0 - Reliabilty and validity not yet determined. Not ready for general use. Question 7. A

A beaker of still water sits on a lab bench. An engineering student adds one drop of blue dye to the water and observes what happens as she stirred the beaker contents.

Eventually, the dye will be uniformly distributed throughout the water and no further change in the distribution of dye will be observed. At this point, we can say:

a. Water and dye are in equilibrium with each other but not at steady-state.

b. The water and dye are at steady-state but are not in equilibrium.

c. Water and dye are in equilibrium and at steady-state.

d. The water and dye are not in equilibrium and are not at steady-state.

because:

e. The net movement of dye in water is zero and there is no liquid flow into or out of the beaker.

f. The net movement of dye in water is zero and all conditions in the beaker (for example, temperature, pressure, volume, concentration) are not changing with time.

g. Diffusion is taking place at the water-dye interface and the rate of dye movement into the water is equal to the rate of water movement into the dye.

h. The beaker is open to the atmosphere, and pressure and temperature could change. 
Version 1.0 - Reliabilty and validity not yet determined. Not ready for general use.

Question 7.B

Water and blue dye are individually and steadily added to a beaker as shown below. The water/dye mixture is steadily removed from the beaker so that the liquid level in the beaker remains constant. The beaker contents are well stirred so that the distribution of dye in the beaker is uniform (same dye concentration at all locations in the beaker contents).

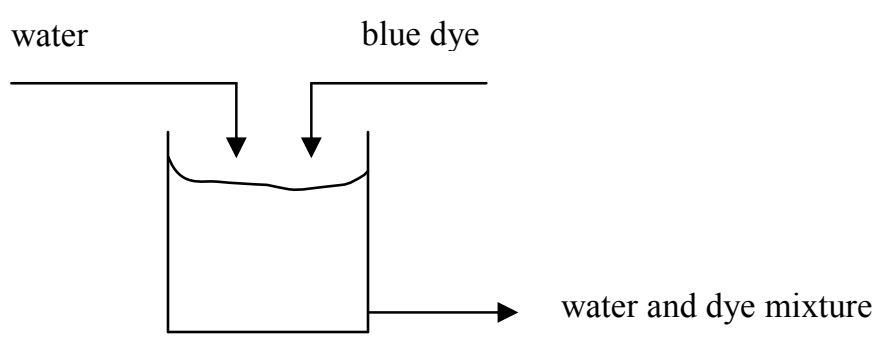

The water and dye flowrates are constant and the total flowrate is 1 liter/minute.

Since the beaker has a volume of 1 liter, the average time water and dye spend in the beaker is 1 minute. If the time required for water and dye to come to equilibrium is 2 minutes, what can we say about the water and dye in the beaker?

a. Water and dye are in equilibrium with each other but not at steady-state.

b. The water and dye are at steady-state but are not in equilibrium.

c. Water and dye are in equilibrium and at steady-state.

d. The water and dye are not in equilibrium and are not at steady-state.

because:

e. The ratio of dye to water is constant throughout the beaker and the liquid flowrate is constant.

f. The water and dye don't have enough time to come to equilibrium but all conditions in the beaker (for example, temperature, pressure, volume, concentration) are not changing with time.

g. The water and dye don't have time to come to equilibrium in the beaker and therefore the system can never be at steady-state.

h. The beaker is open to the atmosphere and pressure and temperature could change. 
Version 1.0 - Reliabilty and validity not yet determined. Not ready for general use. Question 8.

Water is placed between two parallel plates. The bottom plate moves to the right at velocity $\mathrm{V}$ and the top plate is stationary. The water velocity profile shown below is observed at steady-state conditions (you are looking at a side-view of the plates and the water between the plates).

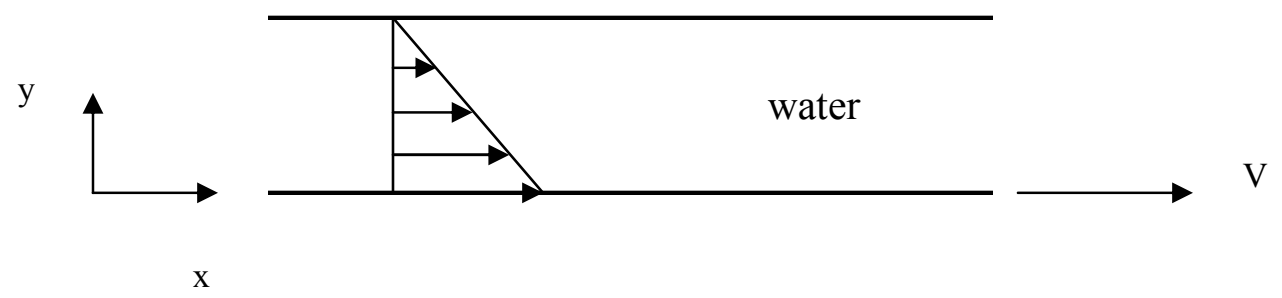

For this flow system, water moves in the x-direction and therefore has x-momentum. In which direction is the $\mathrm{x}$-momentum being transferred?

a. Transfer is in the $-y$ direction (downward in the figure) because as you move in the $-y$ direction the velocity arrows get bigger

b. Transfer is in the $+x$ direction (to the right in the figure) because that's the same direction as the velocity

c. Transfer is in the $+y$ direction (upward in the figure) because momentum is transported from a region of high momentum (near bottom plate) to low momentum (near top plate)

d. Transfer is in the $+x$ direction because the drag force on the plates because of friction is in the direction of the flow

e. Transfer is in the $-x$ direction because momentum transfer and velocity oppose each other 
Version 1.0 - Reliabilty and validity not yet determined. Not ready for general use. Question 9.

Identical rigid vessels contain two different ideal gases as shown below. Vessel 1 contains pure gas "A" while Vessel 2 contains a equimolar mixture of " $A$ " and "B" (same amount of each component in the mixture on a molar basis).

The molecular weight of " $\mathrm{A}$ " is twice that of "B."

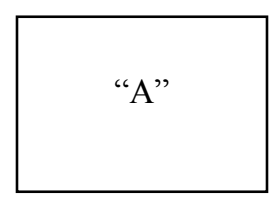

Vessel 1

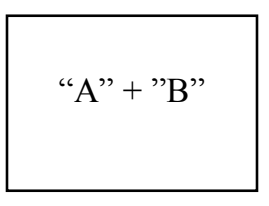

Vessel 2

If the pressure in vessels 1 and 2 is equal $\left(\mathrm{P}_{1}=\mathrm{P}_{2}\right)$ and gas temperature in vessels 1 and 2 is equal $\left(\mathrm{T}_{1}=\mathrm{T}_{2}\right)$, what can be said about the molar density (gmoles/volume) of gas in the two vessels if both gases are ideal?

a. Both have the same molar density since temperature, pressure, and volume are the same.

b. The molar density in vessel 1 is twice the density in vessel 2 because the molecular weight of gas " $\mathrm{A}$ " is twice that of gas "B."

c. The molar density in vessel 1 is half the density in vessel 2 because the molecular weight of gas " $\mathrm{A}$ " is twice that of gas "B."

d. Nothing can be said about molar density because the volume of the vessels is not specified.

e. The molar density of the two gases can never be equal since the molecular weights of " $\mathrm{A}$ " and " $\mathrm{B}$ " are different. 
Version 1.0 - Reliabilty and validity not yet determined. Not ready for general use. Question 10.

A fluid flows at steady-state through a circular pipe of uniform diameter and uniform cross-sectional area. If the outlet density of the fluid decreases to $1 / 2$ of its inlet value, what happens to the fluid average velocity at the outlet?

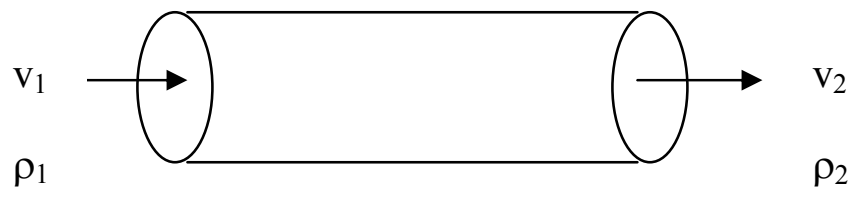

$\mathrm{v}_{1}=$ average fluid velocity at pipe inlet $\quad \mathrm{v}_{2}=$ average fluid velocity at pipe outlet

$\rho_{1}=$ fluid density at pipe inlet $\quad \rho_{2}=$ fluid density at pipe outlet

a. The velocity would not change since mass must be conserved and the flow is steady-state.

b. The outlet velocity will be $1 / 4^{\text {th }}$ of the inlet velocity since kinetic energy must be conserved according to the Bernoulli equation.

c. The outlet velocity will be twice the inlet velocity since mass must be conserved in the system.

d. The velocity will increase a little bit but we can't tell how much with the information given. 